\title{
A cascadic multigrid algorithm for the Stokes equations
}

\author{
Dietrich Braess $^{1}$, Wolfgang Dahmen ${ }^{2}$ \\ 1 Fakultät für Mathematik, Ruhr-Universität, D-44780 Bochum, Germany; \\ e-mail: braess@num.ruhr-uni-bochum.de \\ 2 Institut für Geometrie und Praktische Mathematik, RWTH Aachen, D-52056 Aachen, \\ Germany; e-mail: dahmen@igpm.rwth-aachen.de
}

Received September 10, 1997 / Revised version received February 20, 1998

Summary. A variant of multigrid schemes for the Stokes problem is discussed. In particular, we propose and analyse a cascadic version for the Stokes problem. The analysis of the transfer between the grids requires special care in order to establish that the complexity is the same as that for classical multigrid algorithms.

Mathematics Subject Classification (1991): 65N55, 65N30, 35Q30

\section{Introduction}

Multilevel methods without coarse grid corrections have been defined and applied to elliptic problems of second order by Bornemann and Deuflhard $[1,5]$. They have called it a cascadic algorithm and showed that an optimal iteration method with respect to the energy norm is obtained if conforming elements are used.

Deuflhard's starting point for the cascadic multigrid method [5] was the idea that it should be sufficient to start the iteration at the level $i$ with a good approximation from the level $i-1$. A similar idea can already be found in Chapter 9 of Wachspress' book [12] from 1966, i.e. from the period in which also the first theoretical investigations of multigrid methods were made. The approach from that period had, however, the drawback that not enough steps were performed on the coarse grids. Later Shaidurov [8] established in essence a recursion relation of the form

$$
\left\|u_{i}-v_{i}\right\|_{1} \leq\left\|u_{i-1}-v_{i-1}\right\|_{1}+c \frac{h_{i}}{m_{i}}
$$

Correspondence to: D. Braess

Numerische Mathematik Electronic Edition page 179 of Numer. Math. (1999) 82: 179-191 
for some finite element problems with full regularity. Here $u_{i}$ denotes the exact solution on the level $i$ and $v_{i}$ its approximation computed after $m_{i}$ steps. The accumulation of the error is no problem since the iteration steps on the lower levels are cheap.

It is crucial for the optimality of the algorithm that the error from the previous level enters with a factor of precisely 1 . Since it was not clear whether a constant factor greater than 1 is encountered in the transfer for nonconforming elements, there are no serious conjectures for the latter families. This feature is shared by the Stokes equations as will be obvious in Sect. 4 . In fact, the nonconformity caused by the prolongation operator introduces factors strictly greater than 1 in the recursion (1.1).

There is another difference to classical multigrid algorithms. The recursion relation (1.1) refers only to the energy norm, and it has been proved in [2] that the cascadic version is in general not optimal for the $L_{2}$-norm. This is in contrast to classical multigrid algorithms, see $[7,13]$, where one can more easily move between the $H^{1}$-norm and the $L_{2}$-norm.

We will develop the cascadic multigrid method for saddle point problems which arise from the Stokes problem. Here we will apply the smoothing procedure proposed in [4]. However, prolongating an approximate solution to the next higher level generally destroys the divergence freeness ensured by the smoother. Since the natural correction arising in this context involves a projector that is orthogonal in $L_{2}$ and not with respect to the energy inner product, there is the drawback with the $L_{2}$-norm mentioned above. Nevertheless, we are able to properly isolate the influence of nonconformity and to apply then a duality technique providing sufficiently sharp estimates for the additional terms. This eventually will be shown to yield optimality for our saddle point problems. Since this in turn is related to a careful analysis of the transfer between the grids, the technique is also useful in the treatment of nonconforming elements. ${ }^{1}$ The analysis shows that the loss induced by the transfer between the grids can be controled also in nonstandard cases. This may be of interest for many multigrid algorithms and not only for those of cascadic type.

We note that the cascadic multigrid algorithm offers an efficient alternative to nested iteration for obtaining a good initial guess of the finite element solution. It is not our intention to replace the standard multigrid procedure.

\section{Notation and problem formulation}

Let $\Omega \subset \mathbb{R}^{d}, d=2$ or 3 , and let $H^{s}(\Omega), H_{0}^{s}(\Omega)$ denote the usual Sobolev spaces endowed with the Sobolev norms $\|\cdot\|_{s}$. The space $L_{2,0}(\Omega):=$

\footnotetext{
${ }^{1}$ Note added in proof. Indeed, R. Stevenson [9] told the authors in Jan. 1998 that he has applied the analogous technique to deal with nonconforming elements
}

Numerische Mathematik Electronic Edition page 180 of Numer. Math. (1999) 82: 179-191 
$\left\{q \in L_{2}(\Omega): \int_{\Omega} q d x=0\right\}$ can be identified with $L_{2}(\Omega) / \mathbb{R}$. The weak formulation of the Stokes problem reads: Find $u \in X:=H_{0}^{1}(\Omega)^{d}$ and $p \in M:=L_{2,0}(\Omega)$ such that

$$
\begin{array}{ll}
a(u, v)+b(v, p) & =\langle f, v\rangle \text { for all } v \in X, \\
b(u, q) & =0 \quad \text { for all } q \in M .
\end{array}
$$

Here, $f \in X^{\prime}$, the dual of $X$, is given, with $\langle\cdot, \cdot\rangle$ being the standard duality pairing induced by the $L_{2}$ inner product, and

$$
\begin{aligned}
a(u, v) & :=\int_{\Omega} \nabla u \nabla v d x, \\
b(v, q) & :=-\int_{\Omega} \operatorname{div} v q d x .
\end{aligned}
$$

We assume that the problem is $H^{2}$-regular, e.g. $\Omega$ may be a bounded convex polyhedral domain in 2-space.

We are interested in approximate solutions to (2.1) obtained by finite element discretizations. To this end we assume that for each $i \in \mathbb{N}_{0}, i \leq J$, $T_{i}$ denotes a shape-regular triangulation of $\Omega$ which is generated by successively refining uniformly some initial triangulation $T_{0}$. Shape regularity means that the ratio of the diameter and the radius of the largest inscribed ball of any simplex in $T_{i}$ remains bounded. Accordingly, $X_{i}$ and $M_{i}$ will denote the corresponding conforming finite element spaces of Taylor and Hood $[3,6]$. Likewise we may use any elements with the properties listed in [11]. In particular, the finite element spaces are nested and form an ascending hierarchy of spaces

$$
X_{0} \subset X_{1} \subset \cdots \subset X_{J} \subset X, \quad M_{0} \subset M_{1} \subset \cdots \subset M_{J} \subset M .
$$

Restricting (2.1) to the pair $X_{i}, M_{i}$, gives rise to the linear system of equations

$$
\left(\begin{array}{ll}
A_{i} & B_{i}^{\mathrm{T}} \\
B_{i}
\end{array}\right)\left(\begin{array}{l}
u_{i} \\
q_{i}
\end{array}\right)=\left(\begin{array}{c}
f_{i} \\
0
\end{array}\right)
$$

where as usual the operators $A_{i}, B_{i}$ on $X_{i}$ are for $u_{i} \in X_{i}$ defined by

$$
\left(A_{i} u_{i}, v\right)=a\left(u_{i}, v\right), \quad v \in X_{i}, \quad\left(B_{i} u_{i}, q\right)=b\left(u_{i}, q\right), q \in M_{i} .
$$

Of course, as soon as one fixes bases in $X_{i}$ and $M_{i}$, one obtains matrix representations of $A_{i}, B_{i}$ which will be denoted again by $A_{i}, B_{i}$, respectively. For simplicity we identify the functions $v_{i}, q_{i}$ in $X_{i}, M_{i}$ with their coefficient sequences, always assuming that the bases are normalized so that

$$
\left\|v_{i}\right\|_{0} \sim\left\|v_{i}\right\|_{\ell_{2}} .
$$

Numerische Mathematik Electronic Edition page 181 of Numer. Math. (1999) 82: 179-191 
That is, both norms can be uniformly bounded by constant multiples of each other. Moreover we have the inverse inequalities

$$
\left\|v_{i}\right\|_{1} \leq c h_{i}^{-1}\left\|v_{i}\right\|_{0}, \quad v_{i} \in X_{i} .
$$

Here and throughout the paper $c$ will be a generic constant which is independent of the level and which may be different in different equations.

Our objective is to solve (2.3) for the highest level of resolution $i=J$.

\section{The smoothing operation}

A key ingredient of a multigrid scheme for the solution of (2.3) is a suitable smoother. In the following we will employ the smoother proposed in [4]. Since this can be described for an abstract saddle point problem, for convenience we suppress the subscripts indicating the discretization level. Thus we consider the linear system of equations

$$
\left(\begin{array}{ll}
A & B^{\mathrm{T}} \\
B
\end{array}\right)\left(\begin{array}{l}
u \\
q
\end{array}\right)=\left(\begin{array}{l}
f \\
g
\end{array}\right)
$$

where $A$ is a symmetric positive definite matrix. It characterizes the solution of the constrained minimum problem

$$
\frac{1}{2} u^{\mathrm{T}} A u-f^{\mathrm{T}} u \rightarrow \min ! \text { subject to } B u=g .
$$

Now suppose that $C$ is a preconditioner for $A$ which, in particular, satisfies

$$
v^{\mathrm{T}} A v \leq v^{\mathrm{T}} C v, \quad v \in X,
$$

and for which the linear system

$$
\left(\begin{array}{ll}
C & B^{\mathrm{T}} \\
B
\end{array}\right)\left(\begin{array}{l}
v \\
q
\end{array}\right)=\left(\begin{array}{l}
d \\
e
\end{array}\right)
$$

is more easily solved. Note that the inverse is formally given by

$$
\left(\begin{array}{ll}
C & B^{\mathrm{T}} \\
B
\end{array}\right)^{-1}=\left(\begin{array}{ll}
C^{-1}\left(I-B^{\mathrm{T}} S^{-1} B C^{-1}\right) & C^{-1} B^{\mathrm{T}} S^{-1} \\
S^{-1} B C^{-1} & -S^{-1}
\end{array}\right)
$$

where

$$
S:=B C^{-1} B^{\mathrm{T}}
$$

is the Schur complement of (3.3). Specifically, if $C=\alpha I$, then (3.2) reads

$$
v^{\mathrm{T}} A v \leq \alpha v^{\mathrm{T}} v,
$$

Numerische Mathematik Electronic Edition page 182 of Numer. Math. (1999) 82: 179-191 
i.e., $\alpha$ is assumed to be not smaller than the spectral radius $\rho(A)$ of $A$. In this case (3.4) becomes

$$
\left(\begin{array}{ll}
\alpha I B^{\mathrm{T}} \\
B
\end{array}\right)^{-1}=\left(\begin{array}{lr}
\frac{1}{\alpha} P & B^{\mathrm{T}}\left(B B^{\mathrm{T}}\right)^{-1} \\
\left(B B^{\mathrm{T}}\right)^{-1} B-\alpha\left(B B^{\mathrm{T}}\right)^{-1}
\end{array}\right)
$$

where $P$ is the projection

$$
P:=I-B^{\mathrm{T}}\left(B B^{\mathrm{T}}\right)^{-1} B .
$$

Now, (3.1) is to be solved by an iteration of the form

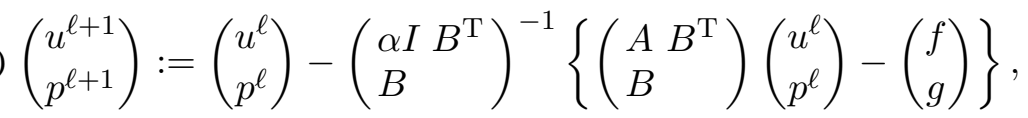

where superscripts will always denote iteration indices. It is important to note that $u^{\ell+1}$ always satisfies the constraint, i.e.,

$$
B u^{\ell+1}=g,
$$

see [4]. Each iteration step requires solving a system of the form (3.3) with $C=\alpha I$. By (3.5), this can be realized by implementing

$$
B B^{\mathrm{T}} q=B d-\alpha e, \quad v=\frac{1}{\alpha}\left(d-B^{\mathrm{T}} q\right) .
$$

Specifically, this amounts to solving an equation similar to the Poisson equation in the case of the Stokes problem. In view of the available efficient Poisson solvers this is acceptable, e.g., smoothers which incorporate Poisson solvers have been used in some efficient multigrid algorithms by Turek [10]. Moreover numerical results in [4] support the expectation that approximate solutions of the equations are sufficient. Obviously it would be against the spirit of the idea of cascadic solvers to use multigrid here, but fortunately there are efficient AMG algorithms which solve the Poisson equation in a black box manner.

In particular, defining for $g=0$

$$
V:=\{v \in X: B v=0\}
$$

the iteration remains in $V$. Therefore one can construct conjugate directions from the corrections in (3.7). In fact, defining the vector

$$
g^{\ell}:=A u^{\ell}+B^{\mathrm{T}} p^{\ell}-f
$$

as the residual of the first block and computing $h^{\ell}$ from

$$
\left(\begin{array}{ll}
\alpha I & B^{\mathrm{T}} \\
B
\end{array}\right)\left(\begin{array}{l}
h^{\ell} \\
p^{\ell}
\end{array}\right)=\left(\begin{array}{c}
g^{\ell} \\
0
\end{array}\right)
$$

Numerische Mathematik Electronic Edition page 183 of Numer. Math. (1999) 82: 179-191 
we obtain the next conjugate direction and the next iterate from

$$
\begin{aligned}
d^{\ell} & :=-h^{\ell}+\beta_{\ell} d^{\ell-1} \\
u^{\ell+1} & :=u^{\ell}+\alpha_{\ell} d^{\ell} .
\end{aligned}
$$

The factors $\alpha_{\ell}$ and $\beta_{\ell}$ are determined as in any cg-algorithm. Note that by construction $B h^{\ell}=0$ so that also

$$
B d^{\ell}=0, \quad \ell=0,1, \ldots
$$

Thus one considers the cg-method confined to a subspace where $A$ is definite. The cg-method based on (3.7) will be employed as a smoother in the cascadic multigrid algorithm in accordance with the concept for scalar equations in $[1,8]$.

\section{The cascadic multigrid iteration}

Our objective is to analyse the following

\section{CASCADIC Multigrid Algorithm:}

Compute the exact solution $u_{0}, q_{0}$ of (2.3) on level $i=0$. Set $v_{0}:=u_{0}$. For $i=1, \ldots, J:\{$

- Compute $w_{i}$ as the prolongation of $v_{i-1}$.

- Compute $v^{0}:=v_{i}^{0}$ as the projection of $w_{i}$ to $V_{i}:=\operatorname{ker} B_{i}$.

- Execute $m=m_{i}$ steps of the cg-method.

- Set $v_{i}:=v^{m}$ \}

Since the spaces $X_{i}, M_{i}$ are nested, the prolongation

$$
v_{i-1} \mapsto w_{i}, \quad q_{i-1} \mapsto q^{0}
$$

in the above scheme is simply the inclusion. However, although each $v_{i-1}^{\ell}$ and hence $v_{i-1}$ belong to $V_{i-1}$, its prolongation $w_{i}$ will generally not belong to $V_{i}$. The correction can be performed by solving the sytem

$$
\left(\begin{array}{ll}
C & B^{\mathrm{T}} \\
B
\end{array}\right)\left(\begin{array}{l}
\tilde{w} \\
\tilde{q}
\end{array}\right)=\left(\begin{array}{c}
0 \\
-B w_{i}
\end{array}\right)
$$

where we again suppress the level index $i$ in the matrices $B, C$. In fact, by the remarks at the beginning of Sect. $3, \tilde{w}$ minimizes the quadratic functional $(C v, v)$ under the constraint $B \tilde{w}=-B w_{i}$. One easily confirms that

$$
\tilde{w}=-C^{-1} B^{\mathrm{T}}\left(B C^{-1} B^{\mathrm{T}}\right)^{-1} B w_{i} .
$$


Hence,

(4.3) $v^{0}:=w_{i}+\tilde{w}=\left(I-C^{-1} B^{\mathrm{T}}\left(B C^{-1} B^{\mathrm{T}}\right)^{-1} B\right) w_{i}=: P_{C} w_{i}$.

Thus, since for $(u, v)_{C}:=(u, C v)=(C v, u)$

$$
\begin{aligned}
\left(P_{C} z-z, w\right)_{C} & =-\left(C^{-1} B^{\mathrm{T}}\left(B C^{-1} B^{\mathrm{T}}\right)^{-1} B z, C w\right) \\
& =\left(\left(B C^{-1} B^{\mathrm{T}}\right)^{-1} B z, B w\right)=0, \quad \text { for all } w \in V_{i},
\end{aligned}
$$

the mapping $P_{C}$ is just the orthogonal projection to $V_{i}$ with respect to the inner product $(\cdot, \cdot)_{C}$.

The most convenient choice for $C$ is $\alpha I$. Noting that $P_{\alpha I}=P_{I}=: P$ (see (3.6)) for any $\alpha>0$ this gives rise to the orthogonal projector with respect to the standard $L_{2}$-inner product, i.e.,

$$
\|P\|_{0}=1 .
$$

For completeness, we note that there is also a bound with respect to the $\|\cdot\|_{1}$-norm.

Lemma 1. For $P=P_{i}$ defined by (3.6) one has $\left\|P_{i}\right\|_{1} \leq c$ uniformly in $i \in \mathbb{N}$.

Proof. It suffices to prove that $S=S_{i}:=B^{\mathrm{T}}\left(B B^{\mathrm{T}}\right)^{-1} B$ is uniformly bounded in $\|\cdot\|_{1}$. To this end, note that $V=\operatorname{ker} B$ is a closed subspace of $H_{0}^{1}(\Omega)^{d}$. Therefore its orthogonal complement $V^{\perp}$ with respect to $(\cdot, \cdot)_{1}$ exists. Thus any $v \in X_{i}$ can be written as $v=z+w$ with $B z=0$ and $w \in V^{\perp}$. Obviously, $B S v=B v=B w$. Hence,

$$
\|S v\|_{1}^{2}=\|S(z+w)\|_{1}^{2}=\|S w\|_{1}^{2} .
$$

On the other hand, since

$$
\|w\|_{1}^{2} \sim(B w, B w) \text { for } w \in V^{\perp},
$$

cf. Remark III.5.5 in [3], we obtain

$$
\|S w\|_{1}^{2} \sim(B S w, B S w)=(B w, B w) \sim\|w\|_{1}^{2} \leq\|w\|_{1}^{2}+\|z\|_{1}^{2}=\|v\|_{1}^{2},
$$

and the assertion follows from (4.5). 


\section{The cg-method and optimal polynomials}

According to (3.8) in [4] the error in the $v$-component for the iteration (3.7) is given by

(5.1) $u-v^{\ell+1}=P\left(I-\frac{1}{\alpha_{\ell}} A\right)\left(u-v^{\ell}\right)=\left(I-\frac{1}{\alpha_{\ell}} P A P\right)\left(u-v^{\ell}\right)$,

where $P$ is defined by (3.6). From the theory of the cg-method we know that $\left\|u-v^{m}\right\|_{1} \leq c\|\| u-v^{m} \|$

$$
=c \inf \left\{\left\|u-Q_{m}(P A P) v^{0}\right\| \mid: \operatorname{deg} Q_{m} \leq m, Q_{m}(0)=1\right\} .
$$

Here the energy norm $\||\cdot| \mid$ is defined by $\|v\| \|:=(v, P A P v)_{0}^{1 / 2}$, so that in the case of the Stokes problem

$$
\left\|\left.v|\|:=| P v\right|_{1} .\right.
$$

It has been shown by Shaidurov [8] that, given $m \in \mathbb{N}$ and $\Lambda>0$, there exists a polynomial $Q_{m}$ such that

$$
\begin{array}{rlrl}
Q_{m}(0) & =1 & \\
\left|\sqrt{x} Q_{m}(x)\right| & \leq \frac{\sqrt{\Lambda}}{2 m+1} & \text { for } x \in[0, \Lambda], \\
\left|Q_{m}(x)\right| & \leq 1 \quad \text { for } x \in[0, \Lambda] .
\end{array}
$$

We set $\lambda_{\max }:=\lambda_{\max }(P A P)$. Since $P A P$ is selfadjoint, following Shaidurov [8] we obtain from (5.4) an operator $Q_{m}$ with

$$
\begin{aligned}
\left\|Q_{m} v\right\| & \leq \frac{\sqrt{\lambda_{\max }}}{2 m+1}\|v\|_{0}, v \in X_{i}, \\
\left\|Q_{m} v\right\| & \leq\|v\| \|, \quad v \in X_{i} .
\end{aligned}
$$

We emphasize that the energy norm is a mesh-dependent norm, since the projector in (5.3) depends on the grid. Therefore we reformulate the above bounds. Since $Q_{m} v$ belongs to $V_{i}$, we have $\left\|\left|Q_{m} v\right|\right\|=\left|Q_{m} v\right|_{1}$, i.e.

$$
\begin{aligned}
& \left|Q_{m} v\right|_{1} \leq \frac{\sqrt{\lambda_{\max }}}{2 m+1}\|v\|_{0}, v \in X_{i}, \\
& \left|Q_{m} v\right|_{1} \leq|P v|_{1}, \quad v \in X_{i} .
\end{aligned}
$$

Although during the computations $Q_{m}$ is only applied to functions in the kernel $V_{i}$, it is crucial for the analysis that the estimates hold for all $v \in X_{i}$. Moreover we note that the inverse inequality (2.5) implies

$$
\lambda_{\max }=\lambda_{\max }(P A P) \leq \lambda_{\max }(A) \leq c h^{-2} .
$$




\section{A recursion relation and final estimates}

In contrast to cascadic iterations for scalar elliptic problems the prolongation of the approximate solution $v_{i-1}$ on level $i-1$ is followed by a correction which projects the prolongated $w_{i}$ to $v^{0}:=P_{i} w_{i} \in V_{i}$. Since $P_{i}$ is an orthogonal projector relative to the $L_{2}$-inner product and therefore generally does not have norm one in $H^{1}$, the relation

$$
\left\|v^{0}-u_{i}\right\|_{1}=\left\|P_{i}\left(w_{i}-u_{i}\right)\right\|_{1}
$$

does not allows us to directly infer the estimate

$$
\left\|v^{0}-u_{i}\right\|_{1} \leq\left\|w_{i}-u_{i}\right\|_{1}
$$

which would be needed in a convergence analysis following the concepts of $[1,8]$. The subsequent discussion indicates the corresponding difficulties. We will overcome them by switching to the $|\cdot|_{1}$-projector in the analysis. The terms which arise from the compensation will be estimated by applying the following lemma.

Lemma 2. There exists a linear mapping $R_{i}: X_{i} \rightarrow V_{i}$ and a constant $c$ such that

$$
\begin{aligned}
\left\|\left(I-R_{i}\right) z_{i}\right\|_{0} & \leq c h_{i}\left\|z_{i}\right\|_{1} \quad \text { for all } z_{i} \in V_{i-1}, \\
\left|R_{i} z_{i}\right|_{1} & \leq\left|z_{i}\right|_{1} \quad \text { for all } z_{i} \in X_{i} .
\end{aligned}
$$

The proof of the lemma will be given in the next section.

First, under the regularity assumption

$$
\|u\|_{2}+\|p\|_{1} \leq c\|f\|_{0},
$$

one obtains the $L_{2}$-estimate

$$
\left\|u-u_{i}\right\|_{0} \leq c h_{i}^{2}\|f\|_{0} .
$$

Thus the triangle inequality yields

$$
\left\|u_{i}-u_{i-1}\right\|_{0} \leq\left\|u_{i}-u\right\|_{0}+\left\|u-u_{i-1}\right\|_{0} \leq c h_{i}^{2}\|f\|_{0} .
$$

Here we assume as usual that $h_{i} / h_{i-1}$ remains bounded.

We are now prepared to analyze the error produced by the scheme described in Sect. 4. To this end, we recall that $u_{i}$ denotes the exact solution of the discrete problem in $X_{i}$, while $v^{0}=v_{i}^{0}:=P_{i} w_{i}$ denotes the starting value

Numerische Mathematik Electronic Edition page 187 of Numer. Math. (1999) 82: 179-191 
for the iteration on the level $i$. As before $w_{i}$ is the prolongation of the approximate solution $v_{i-1}=v^{m_{i-1}}$ in $X_{i-1}$. Abbreviating $Q_{i}:=Q_{m_{i}}\left(P_{i} A_{i} P_{i}\right)$, we obtain

$$
\begin{aligned}
u_{i}-v_{i}= & Q_{i}\left(u_{i}-P_{i} v_{i-1}\right) \\
= & Q_{i}\left(u_{i}-P_{i} u_{i-1}\right)+Q_{i} P_{i}\left(u_{i-1}-v_{i-1}\right) \\
= & Q_{i}\left(u_{i}-P_{i} u_{i-1}\right)+Q_{i} R_{i}\left(u_{i-1}-v_{i-1}\right) \\
& +Q_{i}\left(P_{i}-R_{i}\right)\left(u_{i-1}-v_{i-1}\right) .
\end{aligned}
$$

As for the first summand, we invoke (5.5), (5.7), and (6.3) to obtain

$$
\begin{aligned}
\left|Q_{i}\left(u_{i}-P_{i} u_{i-1}\right)\right|_{1} & \leq c \frac{h_{i}^{-1}}{m_{i}}\left\|u_{i}-P_{i} u_{i-1}\right\|_{0} \\
& \leq c \frac{h_{i}^{-1}}{m_{i}}\left\|u_{i}-u_{i-1}\right\|_{0} \\
& \leq c \frac{h_{i}}{m_{i}}\|f\|_{0} .
\end{aligned}
$$

The second term of (6.4) is estimated by employing (5.6), the estimate (6.2) in Lemma 2, and $P_{i} R_{i}=R_{i}$ :

$$
\begin{aligned}
\left|Q_{i} R_{i}\left(u_{i-1}-v_{i-1}\right)\right|_{1} & \leq\left|P_{i} R_{i}\left(u_{i-1}-v_{i-1}\right)\right|_{1}=\left|R_{i}\left(u_{i-1}-v_{i-1}\right)\right|_{1} \\
& \leq\left|u_{i-1}-v_{i-1}\right|_{1} .
\end{aligned}
$$

The third summand on the right hand side of (6.4) represents the essential distinction from the scalar elliptic case. It reflects the nonconformity of the prolongation. Applying first (5.5), using as before that $P_{i}-R_{i}=P_{i}\left(I-R_{i}\right)$ and bearing (4.4) in mind, provides

$$
\begin{aligned}
\left|Q_{i}\left(P_{i}-R_{i}\right)\left(u_{i-1}-v_{i-1}\right)\right|_{1} & \leq c \frac{h_{i}^{-1}}{m_{i}}\left\|\left(P_{i}-R_{i}\right)\left(u_{i-1}-v_{i-1}\right)\right\|_{0} \\
& \leq c \frac{h_{i}^{-1}}{m_{i}}\left\|\left(I-R_{i}\right)\left(u_{i-1}-v_{i-1}\right)\right\|_{0} .
\end{aligned}
$$

At this point the main estimate (6.1) from Lemma 2 comes into play which yields

$$
\left\|\left(I-R_{i}\right)\left(u_{i-1}-v_{i-1}\right)\right\|_{0} \leq c h_{i}\left|u_{i-1}-v_{i-1}\right|_{1} .
$$

This in turn implies by the previous estimate that

$$
\left|Q_{i}\left(P_{i}-R_{i}\right)\left(u_{i-1}-v_{i-1}\right)\right|_{1} \leq \frac{c}{m_{i}}\left|u_{i-1}-v_{i-1}\right|_{1} .
$$

By combining all three estimates (6.5), (6.6) and (6.7) for the terms on the right hand side of (6.4), we obtain immediately the recursion relation in the following proposition.

Numerische Mathematik Electronic Edition page 188 of Numer. Math. (1999) 82: 179-191 
Proposition 3. There exists a constant c such that

$$
\left|u_{i}-v_{i}\right|_{1} \leq c \frac{h_{i}}{m_{i}}\|f\|_{0}+\left(1+\frac{c}{m_{i}}\right)\left|u_{i-1}-v_{i-1}\right|_{1} .
$$

The choice of $m_{i}$ for the number of smoothing steps on level $i$ was made in [1] such that the term for $i=J$ dominates in the sums $\sum_{i} m_{i}^{-1} h_{i}$ and $\sum_{i} m_{i} h_{i}^{-d}$. (Specifically the choice in [1] corresponds to setting $\alpha:=$ $(d+1) / 2$ in the next theorem.) Therefore the error of the solution $u_{J}$ and the computing effort are given by the contributions of the finest grids.

With the aid of Proposition 3 we are now in a position to establish similar properties here and show that the cascadic multigrid algorithm for the Stokes problem behaves like the cascadic algorithms investigated by Bornemann and Deuflhard [1] and by Shaidurov [8].

Theorem 4. Assume that $1<\alpha<d$ and that the CASCADIC multigrid algorithm described in Sect. 4 is applied with $m_{i}$ cg steps on the levels $1 \leq i \leq J$ the $m_{i}$ being the smallest integers satisfying

$$
m_{i} \geq m_{J} 2^{\alpha(J-i)} .
$$

Then the algorithm yields an approximate solution $v_{J}$ on the highest level with

$$
\left\|u_{J}-v_{J}\right\|_{1} \leq c \frac{h_{J}}{m_{J}}\left\|f_{0}\right\|_{0}
$$

where the constant $c$ is independent of $f$ and J. Moreover, the complexity of the algorithm is bounded by $\mathrm{cm}_{J} \operatorname{dim} X_{J}$.

Proof. Since $v_{0}=u_{0}$, by Proposition 3 we obtain

$$
\left\|u_{J}-v_{J}\right\|_{1} \leq c \sum_{j=0}^{J} \prod_{i=0}^{j-1}\left(1+\frac{c}{m_{J-i}}\right) \frac{h_{J-j}}{m_{J-j}}\|f\|_{0} .
$$

From (6.9) we infer that

$$
\sum_{i=0}^{J-1} \frac{1}{m_{J-i}} \leq \frac{1}{m_{J}} \sum_{i=0}^{J-1} 2^{-\alpha i} \leq \frac{2}{m_{J}} .
$$

Thus the products $\prod_{i=0}^{J-1}\left(1+\frac{c}{m_{J-i}}\right)$ are uniformly bounded by $\exp \left(2 c / m_{J}\right)$ and

$$
\left\|u_{J}-v_{J}\right\|_{1} \leq c \sum_{j=0}^{J} \frac{h_{j}}{m_{j}}\|f\|_{0} .
$$

The estimate (6.10) now follows from (6.11) combined with Lemma 1.3 in [1], while for the above choice of the $m_{i}$ the complexity estimate is a consequence of Lemma 1.4 in [1].

Numerische Mathematik Electronic Edition page 189 of Numer. Math. (1999) 82: 179-191 


\section{Proof of Lemma 2}

Given $z_{i} \in X_{i}$, let $w_{i} \in X_{i}$ be the solution of

$$
\begin{aligned}
a\left(w_{i}, v\right)+b\left(v, p_{i}\right) & =a\left(z_{i}, v\right) \text { for all } v \in X_{i}, \\
b\left(w_{i}, q\right) & =0 \quad \text { for all } q \in M_{i} .
\end{aligned}
$$

Obviously we obtain a linear projection $R_{i}: X_{i} \rightarrow V_{i}$ if we set $R_{i} z_{i}:=w_{i}$.

Since the finite element spaces $X_{i}, M_{i}$ are stable, we have

$$
\left\|w_{i}\right\|_{1}+\left\|p_{i}\right\|_{0} \leq c\left\|z_{i}\right\|_{1} .
$$

In order to apply Nitsche's trick, we consider the auxiliary variational problem

$$
\begin{aligned}
& a(y, v)+b(v, r)=\left(w_{i}-z_{i}, v\right)_{0} \text { for all } v \in H_{0}^{1}(\Omega)^{d}, \\
& b(y, q) \quad=0 \quad \text { for all } q \in L_{2,0}(\Omega) \text {. }
\end{aligned}
$$

Since we have assumed $\mathrm{H}^{2}$-regularity, we obtain

$$
\|y\|_{2}+\|r\|_{1} \leq c\left\|w_{i}-z_{i}\right\|_{0} .
$$

Now we insert $v:=w_{i}-z_{i}$ and $q:=p_{i}$ into (7.3):

$$
\left(w_{i}-z_{i}, w_{i}-z_{i}\right)_{0}=a\left(y, w_{i}-z_{i}\right)+b\left(w_{i}-z_{i}, r\right)+b\left(y, p_{i}\right) .
$$

Note that the left hand side equals $\left\|w_{i}-z_{i}\right\|_{0}^{2}$. Next we recall that (7.1) holds for $v \in X_{i-1}$ and $q \in M_{i-1}$. Since we are only interested in estimates for $z_{i} \in V_{i-1}$, it follows that $b\left(z_{i}, q\right)=0$ for $q \in M_{i-1}$. Hence,

$$
\begin{aligned}
& a\left(w_{i}-z_{i}, v\right)+b\left(v, p_{i}\right)=0 \text { for all } v \in X_{i-1}, \\
& b\left(w_{i}-z_{i}, q\right) \\
& =0 \text { for all } q \in M_{i-1} .
\end{aligned}
$$

The general approximation results for affine families of finite elements [3] guarantee that there is a $y_{i-1} \in X_{i-1}$ such that $\left\|y-y_{i-1}\right\|_{1} \leq c h\|y\|_{2}$ and $r_{i-1} \in M_{i-1}$ such that $\left\|r-r_{i-1}\right\|_{0} \leq c h\|r\|_{1}$. Combining this with (7.5) and (7.6), we obtain

$$
\begin{aligned}
\| w_{i} & -z_{i} \|_{0}^{2} \\
& =a\left(y-y_{i-1}, w_{i}-z_{i}\right)+b\left(w_{i}-z_{i}, r-r_{i-1}\right)+b\left(y-y_{i-1}, p_{i}\right) \\
& \leq c\left\|w_{i}-z_{i}\right\|_{1}\left(\left\|y-y_{i-1}\right\|_{1}+\left\|r-r_{i-1}\right\|_{0}\right)+c\left\|y-y_{i-1}\right\|_{1}\left\|p_{i}\right\|_{0} \\
& \leq c h\left\|w_{i}-z_{i}\right\|_{1}\left(\|y\|_{2}+\|r\|_{1}\right)+c h\|y\|_{2}\left\|p_{i}\right\|_{0} .
\end{aligned}
$$

The triangle inequality and (7.2) yields $\left\|w_{i}-z_{i}\right\|_{1} \leq c\left\|z_{i}\right\|_{1}$. Finally, we use (7.2) for estimating $\left\|p_{i}\right\|_{0}$ and (7.4) for estimating $\|y\|_{2}$ and $\|r\|_{1}$ :

$$
\left\|w_{i}-z_{i}\right\|_{0}^{2} \leq \operatorname{ch}\left\|z_{i}\right\|_{1}\left\|w_{i}-z_{i}\right\|_{0} .
$$

We divide (7.7) by $\left\|w_{i}-z_{i}\right\|_{0}$, set $R_{i} z_{i}:=w_{i}$, and the proof of (6.1) is complete.

In order to prove (6.2) we set $v:=w_{i}, q:=p_{i}$ in (7.1) and obtain

$$
\left|w_{i}\right|_{1}^{2}=a\left(w_{i}, w_{i}\right)=a\left(z_{i}, w_{i}\right)+0 \leq\left|z_{i}\right|_{1}\left|w_{i}\right|_{1} .
$$

After dividing by $\left|w_{i}\right|_{1}$ we have (6.2). 


\section{References}

1. Bornemann, F., Deuflhard, P. (1996): The cascadic multigrid method for elliptic problems. Numer. Math. 75, 135-152

2. Bornemann, F., Krause, R. (1998): Classical and Cascadic multigrid - a methodogical comparison. To appear in: Proceedings of the 9th International Conference on Domain Decomposition, Bjørstad, P., Espedal, M., Keyes, D., eds., John Wiley \& Sons, New York

3. Braess, D. (1997): Finite Elemente. Springer, Berlin-Heidelberg or Finite Elements. Cambridge University Press, Cambridge

4. Braess, D., Sarazin, R. (1997): An efficient smoother for the Stokes Problem. Applied Numer. Math. 23, 3-19

5. Deuflhard, P. (1994): Cascadic conjugate gradient methods for elliptic partial differential equations. Algorithm and numerical results, Proc. 7th Intern. Conf. on Domain Decomposition Methods, Keyes, D., Xu, J., eds., pp. 29-42, AMS, Providence, RI

6. Girault, V., Raviart, R.-A. (1986): Finite Element Methods for Navier-Stokes Equations. Springer-Verlag, Berlin-Heidelberg-New York

7. Hackbusch, W. (1985): Multi-Grid Methods and Applications. Springer-Verlag, BerlinHeidelberg-New York

8. Shaidurov, V.V. (1996): Some estimates of the rate of convergence for the cascadic conjugate gradient method. Comp. Math. Applic. 31(4/5), 161-171

9. Stevenson, R. (1998): The Cascade iteration for nonconforming discretizations. Report 9803 University of Nijmegen

10. Turek, S. (1997): On discrete projection methods for the incompressible Navier-Stokes equations: An algorithmic approach. Comput. Methods Appl. Mech. Engrg. 143, 271288

11. Verfürth, R. (1984): A multilevel algorithm for mixed problems. SIAM J. Numer. Anal. 21, 264-271

12. Wachspress, E.L. (1966): Iterative Solution of Elliptic Systems. Prentice-Hall, Englewood Cliffs, N.J.

13. Yserentant, H. (1993): Old and new convergence proofs for multigrid methods. Acta Numerica 1993, 285-326

Numerische Mathematik Electronic Edition page 191 of Numer. Math. (1999) 82: 179-191 